\title{
LINEAR SLICES OF THE QUASI-FUCHSIAN SPACE OF PUNCTURED TORI
}

\author{
YOHEI KOMORI AND YASUSHI YAMASHITA \\ This paper is dedicated to Professor Caroline Series \\ on the occasion of her 60th birthday.
}

\begin{abstract}
After fixing a marking $(V, W)$ of a quasi-Fuchsian punctured torus group $G$, the complex length $\lambda_{V}$ and the complex twist $\tau_{V, W}$ parameters define a holomorphic embedding of the quasi-Fuchsian space $\mathcal{Q F}$ of punctured tori into $\mathbf{C}^{2}$. It is called the complex Fenchel-Nielsen coordinates of $\mathcal{Q F}$. For $c \in \mathbf{C}$, let $\mathcal{Q}_{\gamma, c}$ be the affine subspace of $\mathbf{C}^{2}$ defined by the linear equation $\lambda_{V}=c$. Then we can consider the linear slice $\mathcal{L}_{c}$ of $\mathcal{Q F}$ by $\mathcal{Q F} \cap \mathcal{Q}_{\gamma, c}$ which is a holomorphic slice of $\mathcal{Q F}$. For any positive real value $c, \mathcal{L}_{c}$ always contains the so-called Bers-Maskit slice $\mathcal{B} M_{\gamma, c}$ defined in [Topology 43 (2004), no. 2, 447-491]. In this paper we show that if $c$ is sufficiently small, then $\mathcal{L}_{c}$ coincides with $\mathcal{B} M_{\gamma, c}$ whereas $\mathcal{L}_{c}$ has other components besides $\mathcal{B} M_{\gamma, c}$ when $c$ is sufficiently large. We also observe the scaling property of $\mathcal{L}_{c}$.
\end{abstract}

\section{INTRODUCTION}

The quasi-Fuchsian space $\mathcal{Q F}$ of once punctured tori can be embedded in $\mathbf{C}^{2}=$ $\{(\lambda, \tau)\}$ by the complex Fenchel-Nielsen coordinates (cf. [5, 9, 15, 16]). By varying the complex twist $\tau$ and keeping the complex length $\lambda$ being fixed as a positive real value $c$, we can define the linear slice $\mathcal{L}_{c} \subset \mathbf{C}$ of $\mathcal{Q F}$. In this paper we investigate the global properties of $\mathcal{L}_{c}$ realized in the complex plane. To state our results, recall that $\mathcal{L}_{c}$ has a component containing the open interval $(2,+\infty)$ which was studied in [5] and also in [7, 15]. In this paper we call this component the standard component and the others non-standard. We will show the following.

Theorem 5.1. There exists some positive constant $c_{0}$ such that for any $c$ satisfying $0<c<c_{0}, \mathcal{L}_{c}$ coincides with the standard component.

Theorem 6.1. There exists some positive constant $c_{1}$ such that for any $c$ satisfying $c>c_{1}, \mathcal{L}_{c}$ contains non-standard components.

In section 7 , we also consider the scaling property of $\mathcal{L}_{c}$.

Corollary 7.3. Linear slice has an asymptotic scaling constant.

See Figure 1 for theorem 5.1 and Figures 2 and 3 for theorem 6.1 and corollary 7.3. The parameters used in the figures are explained in 4.1.

Received by the editors November 7, 2011.

2010 Mathematics Subject Classification. Primary 30F40; Secondary 20H10, 57M50.

The first author was partially supported by Grant-in-Aid for Scientific Research (C) (19540194), Ministry of Education, Science and Culture of Japan. 
Let us describe some historical background of our subject. A marked quasiFuchsian punctured torus group $G$ is a free marked two generator discrete subgroup of $\mathrm{PSL}_{2}(\mathbf{C})$ such that the commutator of the generators is parabolic, and the regular set $\Omega$ consists of two non-empty simply connected invariant components $\Omega^{ \pm}$. Quasi-Fuchsian space $\mathcal{Q F}$ is the space of marked quasi-Fuchsian punctured torus groups modulo conjugation in $P S L_{2}(\mathbf{C})$. The convex core $\mathcal{C} / G$ has two boundary components $\partial \mathcal{C}^{ \pm} / G$ each of which is a once-punctured torus and admits an intrinsic hyperbolic structure making it a pleated surface.

In their seminal paper [5], L. Keen and C. Series defined the Bers-Maskit slice $\mathcal{B} M_{\mu, c}$ for a fixed measured lamination $\mu$ and $c>0$, as the subset of $\mathcal{Q F}$ on which the bending lamination of $\partial \mathcal{C}^{-} / G$ and $\mu$ belong to the same projective class and the length of $\mu$ in $\partial \mathcal{C}^{-} / G$ is equal to $c$. By using their theory of pleating coordinates, they showed that $\mathcal{B} M_{\mu, c}$ is simply connected. J. Parker and J. Parkkonen also studied these slices for the case where $\mu$ is a rational lamination (they call them the $\lambda$-slices), and considered a generalization of I. Kra's plumbing construction and degeneration of $\mathcal{B} M_{\mu, c}$ to the Maskit slice $\mathcal{M}$ (cf. [15]). The first author and J. Parkkonen further studied $\mathcal{B} M_{\mu, c}$; they showed that the boundary of $\mathcal{B} M_{\mu, c}$ is a Jordan curve which is cusped at a countable dense set of points (cf. [7]). In this paper we study the outside of $\mathcal{B} M_{\mu, c}$ in $\mathcal{L}_{c}$ and its scaling property.

This paper is organized as follows. In section 2 we will review the basic notions of the quasi-Fuchsian space $\mathcal{Q F}$ of once punctured tori and its pleating varieties following [5]. The complex Fenchel-Nielsen coordinates of $\mathcal{Q F}$ will be introduced in section 3, and we will define the main subject of this paper, the linear slice $\mathcal{L}_{c}$ of $\mathcal{Q F}$ in section 4 . In sections 5 and 6 we will study connected components of $\mathcal{L}_{c}$ and prove our main theorems. In the last section we will observe the asymptotic self-similarity of $\mathcal{L}_{c}$.

\section{The QUasi-Fuchsian SPaCe $\mathcal{Q} \mathcal{F}$ And Rational Pleating VARIEties}

\subsection{Punctured torus groups and their pleating data.}

2.1.1. Marking. Let $S$ be an oriented once-punctured torus. Any pair of simple closed loops on $S$ that intersect exactly once are free generators of $\pi_{1}(S)$. Let $(\alpha, \beta)$ be such an ordered pair of free generators, chosen so that their commutator $\alpha \beta \alpha^{-1} \beta^{-1}$ represents a positively oriented loop around the puncture. The ordered pair $(\alpha, \beta)$ is called a marking.

2.1.2. $\mathcal{Q} \mathcal{F}$ and $\mathcal{F}$. A punctured torus group is a discrete subgroup $G \subset P S L(2, \mathbf{C})$ that is the image of a faithful representation $\rho$ of $\pi_{1}(S)$ such that the image of the loop around the puncture is parabolic. If $(\alpha, \beta)$ is a marking of $S$, and if $A=\rho(\alpha), B=\rho(\beta)$, then the commutator $K=A B A^{-1} B^{-1}$ is parabolic and the ordered pair $(A, B)=(\rho(\alpha), \rho(\beta))$ is called a marking of $G$.

The group $G$ is quasi-Fuchsian if the regular set $\Omega(G)$ consists of two non-empty simply connected invariant components $\Omega^{ \pm}(G)$. The limit set $\Lambda(G)$ is topologically a circle. Quasi-Fuchsian space $\mathcal{Q F}$ is the space of marked quasi-Fuchsian punctured torus groups modulo conjugation in $\operatorname{PSL}(2, \mathbf{C})$; it has a holomorphic structure induced from the natural holomorphic structure of $S L(2, \mathbf{C})$. 
Let $\mathcal{R}\left(\pi_{1}(S)\right)$ be the set of $P S L(2, \mathbf{C})$-conjugacy classes of representations $\rho$ of $\pi_{1}(S)$ such that the image of the loop around the puncture is parabolic. Considering the compact open topology on $\mathcal{R}\left(\pi_{1}(S)\right)$, Minsky showed that the closure of $\mathcal{Q F}$ in $\mathcal{R}\left(\pi_{1}(S)\right)$ is equal to $\mathcal{D}\left(\pi_{1}(S)\right)$, the set of punctured torus groups modulo conjugation in $P S L(2, \mathbf{C})$ (cf. 13$)$.

Fuchsian space $\mathcal{F}$ is the subset of $\mathcal{Q} \mathcal{F}$ such that the components $\Omega^{ \pm}$are round disks. It is canonically isomorphic to the Teichmüller space of marked conformal structures on $S$.

The quotients $\Omega^{ \pm}(G) / G$ are punctured tori with conformal structures, and hence also with orientations inherited from $\hat{\mathbf{C}}$; we assume that the orientations of $\Omega^{+}(G) / G$ and $S$ agree, whereas those of $\Omega^{-}(G) / G$ and $S$ are opposite.

A point $q \in \mathcal{Q \mathcal { F }}$ represents an equivalence class of marked groups in $P S L(2, \mathbf{C})$. We choose once and for all a triple of distinct points in $\hat{\mathbf{C}}$ and let $G=G(q)$ denote the representative normalized so that the repelling and attracting fixed points of $A$ and the fixed point of $K$ are equal to the fixed triple points in $\hat{\mathbf{C}}$. If it is clear from the context, for readability, we suppress the dependence on $q$.

\subsection{Simple closed curves.}

2.2.1. Enumeration. Denote by $C(S)$, the set of free unoriented homotopy classes of simple closed non-boundary parallel curves on $S$. As is well known, this set can be naturally identified with $\hat{\mathbf{Q}}=\mathbf{Q} \cup \infty$. One way to see this is as follows; let $\mathbf{L}$ denote the integer lattice $m+i n, m, n \in \mathbf{Z} \subset \mathbf{C}$. Topologically, $S$ is the quotient of the punctured plane $\mathbf{C}_{i}=\mathbf{C}-\mathbf{L}$ by the natural action of $G_{i}=\left\langle\hat{A}, \hat{B}_{i}\right\rangle \equiv \mathbf{Z}^{2}$ by the horizontal and vertical translations. A straight line of rational slope in $\mathbf{C}-\mathbf{L}$ projects onto a simple closed curve on the marked punctured torus $S_{i}=\mathbf{C}_{i} / G_{i}$, and the projection of all lines of the same rational slope with the same orientation are homotopic. We denote the unoriented homotopy class obtained by projecting the line of slope $-q / p$ by $[L(p / q)]$. Relative to our choice of marking, $[L(p / q)]$ is in the homology class of $\alpha^{-p} \beta^{q}$ or $\alpha^{p} \beta^{-q}$ on $S_{i}$, where $\alpha, \beta$ are projections of the horizontal and vertical lines corresponding to $\hat{A}, \hat{B}$ respectively. Setting $1 / 0=\infty$, we obtain that the map $\hat{\mathbf{Q}} \rightarrow C(S)$ defined by $p / q \mapsto[L(p / q)]$ which is well defined and bijective. The reason for the choice of convention that $[L(p / q)]$ corresponds to $\alpha^{-p} \beta^{q}$, is the following; if we identify the Teichmüller space Teich $(S)$ of once punctured tori with the upper half plane $\mathbf{H}$, then one can easily compute that the boundary point $p / q \in \hat{\mathbf{R}}$ is the point where the extremal length of curves in the class $[L(p / q)]$ has shrunk to zero.

2.2.2. Special word $W_{p / q}$. Suppose that $\rho: \pi_{1}(S) \rightarrow G \subset P S L_{2}(\mathbf{C})$ is a quasiFuchsian punctured torus group, marked as usual by generators $A=\rho(\alpha), B=$ $\rho(\beta)$. We denote the unique geodesic in the homotopy class of $\rho([L(p / q)])$ in $\mathbf{H}^{3} / G$ by $\gamma_{p / q}$. In particular, for $q \in \mathcal{Q F}, \gamma_{p / q}(q)$ represents the corresponding geodesic in $\mathbf{H}^{3} / G(q)$.

For each $p / q \in \hat{\mathbf{Q}}$, we can find an explicit word $W_{p / q}$ in the marked generators $\langle\alpha, \beta\rangle$ of $\pi_{1}(S)$ representing $[L(p / q)]$ as follows. The words are generated from the initial data

by the formula

$$
W_{0 / 1}=\beta, W_{1 / 0}=\alpha^{-1}
$$

$$
W_{(p+r) /(q+s)}=W_{r / s} W_{p / q},
$$


whenever $p / q<r / s$ and $p s-q r=-1$. We denote by $W_{p / q}(q)$ the corresponding special word in $G(q)$.

\subsection{Rational pleating varieties.}

2.3.1. The pleating loci. We are now ready to discuss the convex hull boundary and the pleating locus. Let $q \in \mathcal{Q F}$ and let $G=G(q)$ be the corresponding marked quasi-Fuchsian group with the regular set and the limit set $\Omega(G), \Lambda(G)$ respectively. The 3 -manifold $\mathbf{H}^{3} / G$ is homeomorphic to $S \times(0,1)$. The surfaces $\Omega(G) / G$ at infinity form the boundary $S \times\{0,1\}$. Let $\partial \mathcal{C}(G)$ be the boundary of the hyperbolic convex hull of $\Lambda(G)$ in $\mathbf{H}^{3}$; it is clearly invariant under the action of $G$. The nearest point retraction $\Omega(G) \rightarrow \partial \mathcal{C}(G)$, defined by mapping $x \in \Omega(G)$ to the unique point of contact with $\partial \mathcal{C}(G)$ of the largest horoball in $\mathbf{H}^{3}$ centered at $x$ with interior disjoint from $\partial \mathcal{C}(G)$, can easily be modified to a $G$-equivariant homeomorphism. We denote two connected components of $\partial \mathcal{C}(G)$ corresponding to $\Omega^{ \pm}(G)$ by $\partial \mathcal{C}^{ \pm}(G)$ respectively. Thus each component $\partial \mathcal{C}^{ \pm}(G) / G$ is topologically a punctured torus. (In the special case in which $G$ is Fuchsian, $\partial \mathcal{C}(G)$ is a flat plane whose two sides serve as a substitute for the two components $\partial \mathcal{C}^{ \pm}(G)$.)

$\partial \mathcal{C}^{ \pm}(G) / G$ are pleated surfaces in $\mathbf{H}^{3} / G$. More precisely, there are complete hyperbolic surfaces $S^{ \pm}$, each homeomorphic to $S$, and maps $f^{ \pm}: S^{ \pm} \rightarrow \mathbf{H}^{3} / G$, such that every point in $S^{ \pm}$is in the interior of some geodesic arc which is mapped by $f^{ \pm}$to a geodesic arc in $\mathbf{H}^{3} / G$, and such that $f^{ \pm}$induce isomorphisms $\pi_{1}(S) \rightarrow$ $G$. Further, $f^{ \pm}$are isometries onto their images with the path metric induced from $\mathbf{H}^{3}$ (cf. [17]). The bending or pleating locus of $\partial \mathcal{C}^{ \pm}(G) / G$ consists of those points of $S^{ \pm}$contained in the interior of one and only one geodesic arc which is mapped by $f^{ \pm}$to a geodesic arc in $\mathbf{H}^{3} / G$. For $G$ non-Fuchsian, the pleating loci are geodesic laminations, meaning that they are unions of pairwise disjoint simple closed geodesics on $S^{ \pm}$. We denote these laminations by $\left|p l^{ \pm}(q)\right|$, and usually identify such a lamination with its image under $f^{ \pm}$in $\mathbf{H}^{3} / G$. A geodesic lamination is called rational if it consists entirely of closed leaves. We concentrate on the special case in which at least one of the pleating loci is rational in this sense. Since the maximum number of pairwise disjoint simple closed curves on a punctured torus is one, such a lamination consists of a single simple closed geodesic and is therefore of the form $\gamma_{p / q}(q)$ for some $p / q \in \hat{\mathbf{Q}}$.

2.3.2. Rational pleating varieties and hyperbolic loci. Given $p / q \in \hat{\mathbf{Q}}$, we set

$$
\mathcal{P}_{p / q}^{ \pm}=\left\{q \in \mathcal{Q F}:\left|p l^{ \pm}(q)\right|=\gamma_{p / q}(q)\right\} \text { and } \mathcal{P}_{p / q}=\mathcal{P}_{p / q}^{+} \cup \mathcal{P}_{p / q}^{-} .
$$

We call these sets the $p / q$-pleating varieties.

For any $p / q \in \hat{\mathbf{Q}}$, consider the trace $\operatorname{Tr} W_{p / q}$ of the special word $W_{p / q}$ associated to $p / q$ defined in 2.2.2. For $q \in \mathcal{R}\left(\pi_{1}(S)\right)$, we may consider the function $T_{p / q}(q)=$ $\operatorname{Tr} W_{p / q}(q)$ as a rational function on $\mathcal{R}\left(\pi_{1}(S)\right)$. We define the hyperbolic locus of $T_{p / q}$ to be the set

$$
\mathcal{H}_{p / q}=\left\{q \in \mathcal{R}\left(\pi_{1}(S)\right): T_{p / q}(q) \in \mathbf{R},\left|T_{p / q}(q)\right|>2\right\} .
$$

Then the next result is fundamental (cf. Proposition 22 in [5]).

Proposition 2.1. $\mathcal{P}_{p / q} \subset \mathcal{H}_{p / q}$. 


\section{The complex Fenchel-Nielsen coordinates of $\mathcal{Q F}$}

3.1. The complex length of a loxodromic element. The complex translation length $\lambda_{M} \in \mathbf{C} / 2 \pi i \mathbf{Z}$ of $M \in P S L(2, \mathbf{C})$ is given by the equation

$$
\pm \operatorname{Tr} M=2 \cosh \lambda_{M} / 2
$$

where $\operatorname{Tr} M$ is the trace of $M$ and we choose the sign so that $\Re \lambda_{M} \geq 0$.

The complex length is invariant under conjugation by Möbius transformations and has the following geometric interpretation, provided $M$ is not parabolic; let $x$ be a point on the axis $\operatorname{Ax} M$ of $M$ and let $\bar{v}$ be a vector normal to $\operatorname{Ax} M$ at $x$. Then $\Re \lambda_{M}$ is the hyperbolic distance between $x$ and $M(x)$ and $\Im \lambda_{M}$ is the angle $\bmod 2 \pi$ between $M(\bar{v})$ and the parallel transport of $\bar{v}$ to $M(x)$, measured facing the attracting fixed point $M^{+}$of $M$. In particular, if $M$ is loxodromic, then $\Re \lambda_{M}>0$ and if $M$ is purely hyperbolic, then in addition $\Im \lambda_{M} \in 2 \pi \mathbf{Z}$.

For $q \in \mathcal{Q F}$ and $\gamma \in C(S)$, we denote the element in the group $G(q)$ representing $\gamma$ by $W(q)$. Because the trace is a conjugation invariant, the complex translation length $\lambda_{W}(q)$ depends only on $q$ and is independent of the normalization of $G(q)$. We want to define the complex length $\lambda_{\gamma}(q)=\lambda_{W}(q)$ as a holomorphic function on $\mathcal{Q F}$ with values in $\mathbf{C}$, not $\mathbf{C} / 2 \pi i \mathbf{Z}$. To do this, we choose the branch that is real valued on $\mathcal{F}$. Since $\lambda_{\gamma} \neq 0$ on $\mathcal{Q} \mathcal{F}$, this choice uniquely determines a holomorphic function $\lambda_{\gamma}: \mathcal{Q F} \rightarrow \mathbf{C}$. From now on, the term "complex length" will always refer to this branch.

We remark that $\Re \lambda_{\gamma}(q)$ is the hyperbolic length of $\gamma$ in $\mathbf{H}^{3} / G(q)$.

3.2. The complex Fenchel-Nielsen coordinates. The complex Fenchel-Nielsen parameters were introduced in [9, 16] as a generalization to $\mathcal{Q F}$ of the classical Fenchel-Nielsen coordinates for Fuchsian groups. Here we briefly summarize the main points as applied to the case of a punctured torus $S$.

Let $G=\langle A, B\rangle$ be a marked quasi-Fuchsian punctured torus group constructed from a pair of marked generators $\alpha, \beta$ of $\pi_{1}(S)$ as described in 2.1. The complex Fenchel-Nielsen coordinates $\left(\lambda_{A}, \tau_{A, B}\right)$ for $G=\langle A, B\rangle$ are obtained as follows; the parameter $\lambda_{A} \in \mathbf{C} / 2 \pi i \mathbf{Z}$ is the complex translation length of the generator $A=$ $\rho(\alpha)$, or equivalently the complex length $\lambda_{\alpha}$. The twist parameter $\tau_{A, B} \in \mathbf{C} / 2 \pi i \mathbf{Z}$ measures the complex shear when the axis $\mathrm{Ax}^{-1} A B$ is identified with the axis $\mathrm{Ax} A$ by $B$. More precisely, if the common perpendicular $\delta$ to $\operatorname{Ax} B^{-1} A B$ and $\operatorname{Ax} A$ meets these axes in points $Y, X$ respectively, then $\Re \tau_{A, B}$ is the signed distance from $X$ to $B(Y)$ and $\Im \tau_{A, B}$ is the angle between $\delta$ and the parallel translate of $B(\delta)$ along $\mathrm{Ax} A$ to $X$, measured facing towards the attracting fixed point of $A$. The conventions for measuring the signed distance and the angle are explained in more detail in [4.

As shown in [15, 9, 4, given the parameters $\lambda_{A}, \tau_{A, B}$, and fixed a normalization, one can explicitly write down the matrix generators for a marked two generator group $G\left(\lambda_{A}, \tau_{A, B}\right) \subset P S L(2, \mathbf{C})$ in which the commutator $[A, B]$ is parabolic as follows:

$$
\begin{gathered}
A=\left(\begin{array}{cc}
\cosh \left(\frac{\lambda}{2}\right) & \cosh \left(\frac{\lambda}{2}\right)+1 \\
\cosh \left(\frac{\lambda}{2}\right)-1 & \cosh \left(\frac{\lambda}{2}\right)
\end{array}\right), \\
B=\left(\begin{array}{cc}
\cosh \left(\frac{\tau}{2}\right) \operatorname{coth}\left(\frac{\lambda}{4}\right) & -\sinh \left(\frac{\tau}{2}\right) \\
-\sinh \left(\frac{\tau}{2}\right) & \cosh \left(\frac{\tau}{2}\right) \tanh \left(\frac{\lambda}{4}\right)
\end{array}\right) .
\end{gathered}
$$


This group may or may not be discrete. The matrix coefficients of $G$ depend holomorphically on the parameters. The construction thus defines a holomorphic embedding of $\mathcal{Q} \mathcal{F}$ into a subset of $\mathbf{C} / 2 \pi i \mathbf{Z} \times \mathbf{C} / 2 \pi i \mathbf{Z}$, in which Fuchsian space $\mathcal{F}$ is identified with the image of $\mathbf{R}^{2}$.

We want to lift this to an embedding into $\mathbf{C}^{2}$. In 3.1 we discussed how to lift the length function $\lambda_{A}$ on $\mathcal{Q F}$ to a holomorphic function on $\mathbf{C}$. We can similarly lift the twist parameter $\tau_{A, B}$ by specifying that it will be real valued on $\mathcal{F}$.

On $\mathcal{F}$, the real valued parameters $\lambda_{A}, \tau_{A, B}$ reduce to the classical Fenchel-Nielsen parameters $l_{A}, t_{A, B}$ defined by the above construction with $\lambda_{A}$ the hyperbolic translation length $l_{A}$ of $A$ and $\tau_{A, B}$ the twist parameter $t_{A, B}$.

3.3. Rational quakebends and pleated surfaces. Clearly, the complex FenchelNielsen coordinates can be made relative to any marking $V, W$ of $G$. As described in detail in section 5 of [4], for fixed $\lambda \in \mathbf{R}^{+}$and $\tau \in \mathbf{C}$, the complex Fenchel-Nielsen coordinates relative to $V, W$ determines a pleated surface $\psi: \mathbf{D} \rightarrow \mathbf{H}^{3}$. We review this process.

Write $\mathcal{V}$ for the set of all lifts of the simple closed curve $\gamma$ corresponding to $V$ to D. Since $\gamma$ is simple, $\mathcal{V}$ consists of a set of pairwise disjoint geodesics in $\mathbf{D}$, namely the axis of $V$ and all of its conjugates under $G(\lambda, \Re \tau)$. These axes in $\mathcal{V}$ partition D into pieces $P_{i}$. The map $\psi$ is defined in such a way that $\psi$ is an isometry on each axis in $\mathcal{V}$ and on each closed piece $P_{i}$. Let $x, y \in \mathbf{D}-\mathcal{V}$ and let $\beta$ be an oriented geodesic from $x$ to $y$. Let $P_{0}, P_{1}, \ldots, P_{k}$ be the pieces cut in order by $\beta$, that meet along axes $\alpha_{1}, \alpha_{2}, \ldots, \alpha_{k} \in \mathcal{V}$. Orient $\alpha_{i}$ so that, in $\mathbf{D}, P_{i-1}$ lies to the left of $\alpha_{i}$ and $P_{i}$ to the right. Let $X_{i}=\beta \cap \alpha_{i}$ and let $\bar{v}_{i}, \bar{w}_{i}$ be tangent vectors to $\psi\left(P_{i-1} \cap \beta\right)$ and $\psi\left(P_{i} \cap \beta\right)$ at $\psi\left(X_{i}\right)$, oriented in the direction inherited from $\beta$, so that $\bar{v}_{i}$ points out of $\psi\left(P_{i-1}\right)$ and $\bar{w}_{i}$ points into $\psi\left(P_{i}\right)$. Let $\bar{v}_{i}^{\prime}, \bar{w}_{i}^{\prime}$ be the projections of $\bar{v}_{i}, \bar{w}_{i}$ onto the directions orthogonal to the image of the bending axis at $\psi\left(X_{i}\right)$. Then $\Im \tau$ is the angle from $\bar{v}_{i}^{\prime}$ to $\bar{w}_{i}^{\prime}$ measured facing along $\psi\left(\alpha_{i}\right)$. We embed $\mathbf{D}$ in the hyperbolic ball model $\mathbf{B}^{3}$ of $\mathbf{H}^{3}$ as the equatorial plane such that the origins in $\mathbf{D}$ and in $\mathbf{B}^{3}$ coincide. We arrange that the axes of $V$ and $W V W^{-1}$ in $G(\lambda, \Re \tau)$ lie in the boundary of a piece $P_{0}$ contained in $\mathbf{D}$. We then choose $\psi$ to be the identity on $P_{0}$. We set $\mathbf{D}_{\gamma}(\lambda, \tau)=\psi(\mathbf{D})$ for the image of the pleated surface in $\mathbf{B}^{3}$. Then $\psi$ induces the group isomorphism $\psi_{*}: G(\lambda, \Re \tau) \rightarrow G(\lambda, \tau)$ satisfying that $\psi(g(z))=\psi_{*}(g)(\psi(z))$ for $g \in G(\lambda, \Re \tau)$ and $z \in \mathbf{D}$.

The next proposition explains the relation between $\psi$ and the bending locus of $\partial \mathcal{C}^{-}(G(q))$ for $q \in \mathcal{Q F}$.

Proposition 3.1. For $q \in \mathcal{Q F}$, let $(\lambda, \tau)$ be the complex Fenchel-Nielsen coordinates relative to marked generators $(V, W)$ of $G(q)$, and let $\gamma$ be the simple closed curve corresponding to $V$. Assume that $V$ is purely hyperbolic and let $\psi: \mathbf{D} \rightarrow \mathbf{H}^{3}$ be the pleated surface defined above. Then $\psi$ is a homeomorphism if and only if $\left|p l^{ \pm}(q)\right|=\gamma$.

Proof. First suppose that $\psi$ is a homeomorphism. Then the boundary of $\mathbf{D}_{\gamma}(\lambda, \tau)$ is $\Lambda(G(q))$. $\mathbf{D}_{\gamma}(\lambda, \tau)$ divides $\mathbf{H}^{3}$ into two domains; one of which is convex, hence contains $\mathcal{C}(G(q))$. Moreover, $\mathbf{D}_{\gamma}(\lambda, \tau)$ contains the axis of $V$ and all of its conjugates in $G(q)$, and the complement of them consist of totally geodesic pieces. Therefore, it is one of the components of $\partial \mathcal{C}(G(q))$ (cf. section 7.1 in [4]).

Next suppose that $\left|p l^{ \pm}(q)\right|=\gamma$. Then $\partial \mathcal{C}^{ \pm}(G(q))$ consists of the axis of $V$ and all of its conjugates in $G(q)$, and totally geodesic pieces. The stabilizer subgroup 
of each totally geodesic piece is conjugate to the Fuchsian subgroup $\left\langle V, W V W^{-1}\right\rangle$. Therefore we can construct the pleated surface satisfying $\mathbf{D}_{\gamma}(\lambda, \tau)=\partial \mathcal{C}^{ \pm}(G(q))$, which implies that $\psi$ is a homeomorphism.

3.4. Rational quakebend planes. Let $\left(\lambda_{V}, \tau_{V, W}\right) \subset \mathbf{C}^{2}$ be the complex FenchelNielsen coordinates relative to marked generators $(V, W)$ of $G$, and let $\gamma$ be the simple closed curve corresponding to $V$. Assume that $V$ is purely hyperbolic and let $c$ be the hyperbolic length of $\gamma$ in $\mathbf{H}^{3} / G$.

We denote the slice $\left\{(c, \tau) \in \mathbf{C}^{2} \mid \tau \in \mathbf{C}\right\}$ by $\mathcal{Q}_{\gamma, c}$ and call it the rational quakebend plane.

Clearly, $\mathcal{Q}_{\gamma, c}$ meets $\mathcal{F}$ along the earthquake path (cf. [5]). The quakebend parameter $\tau$ is a holomorphic coordinate on $\mathcal{Q}_{\gamma, c}$.

On $\mathcal{Q}_{\gamma, c}$, the quakebend parameter $\tau$ and $\operatorname{Tr} W$ are related by

$$
\operatorname{Tr} W=2 \operatorname{coth}\left(\frac{c}{2}\right) \cosh \left(\frac{\tau}{2}\right) .
$$

On $\mathcal{Q}_{\gamma, c}, \operatorname{Tr} W$ is a holomorphic function of $\tau$, branched at $\tau=2 \pi i n(n \in \mathbf{Z})$ (see figure 5.1 in [15]). When $\operatorname{Tr} V$ is real, $\mathcal{Q F} \cap \mathcal{Q}_{\gamma, c}$ is contained in the strip

$$
\{\tau \in \mathbf{C} \mid-\pi i<\Im \tau<\pi i\}
$$

from the argument in 3.3 . Tr $W$ takes the right half strip

$$
\{\tau \in \mathbf{C} \mid \Re \tau>0,-\pi i<\Im \tau<\pi i\}
$$

conformally onto the right half plane $\mathbf{C}^{+}$minus the interval $\left(0,2 \operatorname{coth}\left(\frac{c}{2}\right)\right]$ where the interval $\{\tau \in \mathbf{C} \mid \Re \tau=0,-1<\Im \tau<1\}$ in the imaginary axis is folded at the origin by $\operatorname{Tr} W$ and its image is $\left(0,2 \operatorname{coth}\left(\frac{c}{2}\right)\right]$. We remark that $\mathcal{Q} \mathcal{F} \cap \mathcal{Q}_{\gamma, c}$ is also periodic under the action of the Dehn twist $(A, B) \mapsto\left(A, A^{n} B\right)$, and symmetric under the holomorphic involution $\tau \mapsto-\tau$.

\section{The Linear SLiCE $\mathcal{L}_{c}$}

4.1. Definition. For $q \in \mathcal{R}\left(\pi_{1}(S)\right)$, a marked group $G(q)=\langle A, B\rangle$ modulo conjugation in $P S L(2, \mathbf{C})$ is uniquely determined by $\operatorname{Tr} A, \operatorname{Tr} B$ and $\operatorname{Tr} A B$. In fact, ignoring marking, $G(q)$ modulo conjugation in $P S L(2, \mathbf{C})$ is determined only by $\operatorname{Tr} A$ and $\operatorname{Tr} B$ (more precisely, the pair $(\operatorname{Tr} A, \operatorname{Tr} B$ ) determines a marked group $\langle A, B\rangle$ or $\left\langle A, B^{-1}\right\rangle$ modulo conjugation in $\left.P S L(2, \mathbf{C})\right)$. As an application of the Jorgensen's theory on the combinatorial structure of the Ford domain of a punctured torus group, there is an algorithm roughly answering whether $G(q)$ is a geometrically finite discrete group or not from the data ( $\operatorname{Tr} A, \operatorname{Tr} B$ ) (cf. [1]). Especially fixing $\operatorname{Tr} A=c$, then we can use this algorithm to draw the picture of

$$
\mathcal{D}_{c}=\left\{\operatorname{Tr} B \in \mathbf{C}^{+} \mid G(q)=\langle A, B\rangle \text { is a geometrically finite discrete group }\right\} .
$$

We call this set the discrete locus. Let $\mathcal{Q}_{\gamma, c}$ be the rational quakebend plane in the complex Fenchel-Nielsen coordinates relative to the corresponding marked generators $(A, B)$ of $G$ where we assume that $c=\lambda_{A}(q)$ is real. The linear slice $\mathcal{L}_{c}$ in the right half plane $\mathbf{C}^{+}$, which is the image of $\mathcal{Q} \mathcal{F} \cap \mathcal{Q}_{\gamma, c}$ under $\operatorname{Tr} B$. Because $\mathcal{Q F}$ is open in $\mathbf{C}^{2}$ in complex Fenchel-Nielsen coordinates and $\operatorname{Tr} B$ is an open map on $\mathcal{Q}_{\gamma, c}, \mathcal{L}_{c}$ is open in $\mathbf{C}^{+}$. Then from the definition of $\mathcal{D}_{c}$ and $\mathcal{L}_{c}, \mathcal{L}_{c}$ is a subset of the interior of $\mathcal{D}_{c}$. 
Proposition 4.1. The interior of $\mathcal{D}_{c}$ is equal to $\mathcal{L}_{c}$.

Proof. It is enough to show that any point $q_{0}$ of $\mathcal{D}_{c}$, not contained in $\mathcal{L}_{c}$ is a boundary point of $\mathcal{D}_{c}$. First suppose that $G\left(q_{0}\right)$ is not a free group. Then some word, say $g\left(q_{0}\right)$ is trivial in $G\left(q_{0}\right)$. Then applying the Jorgensen's inequality for the subgroup $H\left(q_{0}\right)$ generated by $g$ and $K=[A, B]$, we can see that if we take a small neighborhood $U$ of $q_{0}$, for any point $q$ of $U$ except $q_{0}, H(q)$ is not discrete which means that $G(q)$ is also indiscrete. Therefore $q_{0}$ is an isolated point of $\mathcal{D}_{c}$. Next, suppose that $G\left(q_{0}\right)$ is free, hence a geometrically finite non-quasi-Fuchsian punctured torus group. Then it must be a cusp (cf. [13]). Hence there is some word, say $g(q)$ which is parabolic in $G\left(q_{0}\right)$. Since $\operatorname{Tr} g$ is a holomorphic function of $\operatorname{Tr} B$, it is an open map; hence, there is a path in the $\operatorname{Tr} B$-plane starting from $q_{0}$ such that on this path, $g$ is elliptic. Therefore, this path is outside of $\mathcal{D}_{c}$. This implies that $q_{0}$ is a boundary point of $\mathcal{D}_{c}$.

From this result, we can see $\mathcal{L}_{c}$ as the interior $\mathcal{D}_{c}$ and study them experimentally. Figures at the end of this paper, drawn by the second author, show computer-generated linear slices revealing some global properties. The black region corresponds to the discrete locus. In the first picture, $\operatorname{Tr} A$ is fixed at 2 and $\operatorname{Tr} B$ ranges in the square of width 4 centered at $\operatorname{Tr} B=2$ so that we see the familiar picture of the Maskit slice. By setting $\operatorname{Tr} A=2.5$, we get the second picture. In Figures 2 and 3, the value of $\operatorname{Tr} A$ is fixed at 8 and 100, respectively, while changing the ranges of $\operatorname{Tr} B$. The width of the squares are 16, 32, 128 and 128, 2560, 12800 , respectively. We can clearly see the "rough self-similarity" of the pictures between Figures 3 and 5 and between Figures 6 and 8, which we will discuss in section 7 .

\subsection{Connected components of $\mathcal{L}_{c}$.}

Proposition 4.2. For any $c>0, \mathcal{L}_{c}$ has a component containing an open interval $(2,+\infty)$.

Proof. There exists a component in $\mathcal{Q F} \cap \mathcal{Q}_{\gamma, c}$ containing $\mathcal{F} \cap \mathcal{Q}_{\gamma, c}$ the real line which is periodic under the action of the Dehn twist $B \mapsto A^{n} B$ and symmetric under $\tau \mapsto-\tau$ (cf. [5, 15], see also [11]). Then its image under $\operatorname{Tr} B$ is the required component.

This component is called the BM-slice in [5] and also called the $\lambda$-slice in [15]. In this paper we call this component of $\mathcal{L}_{c}$ the standard component, and call the other components the non-standard components if they exist. Because of the existence of the standard component which contains the critical value of $\operatorname{Tr} B$, if there is a non-standard component, it is a conformal image of a component of $\mathcal{Q F} \cap \mathcal{Q}_{\gamma, c}$ under the map $\operatorname{Tr} B$. Therefore, we can consider that $\mathcal{L}_{c}$ describes the picture of $\mathcal{Q F} \cap \mathcal{Q}_{\gamma, c}$. The next result shows that topologically every component is a disk.

Proposition 4.3. Each component of $\mathcal{L}_{c}$ is simply connected.

Proof. This is a consequence of a result of McMullen 11 that $\mathcal{Q F}$ is disk convex in $\mathcal{R}\left(\pi_{1}(S)\right)$; that is, for any continuous map $f: \bar{\Delta} \rightarrow \mathcal{R}\left(\pi_{1}(S)\right)$ whose restriction to the unit disk $\Delta$ is holomorphic, $f(\partial \bar{\Delta}) \subset \mathcal{Q F}$ implies $f(\bar{\Delta}) \subset \mathcal{Q F}$.

Remark 4.4 (The Maskit slice). If we consider the limiting case where $c=0$, we can no longer consider the complex Fenchel-Nielsen coordinates. But by using $\operatorname{Tr} B$, 
we can realize the part of the boundary of $\mathcal{Q F}$ defined by the condition that $A$ is parabolic. Then the standard component defined above corresponds to the so-called Maskit slice $\mathcal{M}$ (cf. [3]).

\section{NON-EXISTENCE OF NON-STANDARD COMPONENTS}

\subsection{Otal's result.}

Theorem 5.1. There is some positive constant $c_{0}$ such that for any $c$ satisfying $0<c<c_{0}, \mathcal{L}_{c}$ coincides with the standard component.

This is an immediate consequence of the following result due to J. P. Otal [14].

Theorem 5.2 (cf. corollaire 9.1 in [14]). There exists a positive constant $c_{0}$ such that for a marked quasi-Fuchsian punctured torus group $G(q)$ and $V \in G(q)$ representing a simple closed geodesic $\gamma$ in $\mathbf{H}^{3} / G(q)$, if $V$ is purely hyperbolic and the hyperbolic length $\lambda_{\gamma}(q)$ of $\gamma$ is less than $c_{0}$, then $\gamma$ is a bending locus of $\partial \mathcal{C}(G(q))$.

Following the proof of proposition 9 in [14, we will give a proof of theorem 5.2 to estimate $c_{0}$ in the next subsection 5.2.

Suppose that $\gamma$ is not the bending locus of $\partial \mathcal{C}(G(q))$. Then the pleated surface $\psi$ is not a homeomorphism by proposition 3.1. Let $H$ be a Fuchsian subgroup $\left\langle V, W V W^{-1}\right\rangle$ where $(V, W)$ is a marking of $G(q)$. Denote the totally geodesic plane whose boundary $\partial \mathbf{D}$ contains $\Lambda(H)$ by $\mathbf{D} \subset \mathbf{H}^{3}$. Let $P$ be the convex hull of $\Lambda(H)$ in $\mathbf{D} \subset \mathbf{H}^{3}$. Then $g(P)$ is the convex hull of $\Lambda\left(g H g^{-1}\right)$ in $g(\mathbf{D}) \subset \mathbf{H}^{3}$. Now we have a following claim.

Proposition 5.3. If the pleated surface $\psi$ is not a homeomorphism, then there exists $g \in G(q)$ such that $P$ and $g(P)$ intersect transversally in the axis of $V$.

To show this proposition, we need two lemmas.

Lemma 5.4. For $g \in G(q), g H g^{-1} \cap H$ is a trivial or cyclic subgroup generated by $g V g^{-1}$.

Lemma 5.5. For $g \in G(q), \Lambda\left(g H g^{-1}\right) \cap \Lambda(H)$ is empty or has fixed points of $g V g^{-1}$.

Proof. $H$ and $\mathrm{gHg}^{-1}$ are Fuchsian subgroups of a quasi-Fuchsian group $G(q)$. From a theorem of Suskind (see theorem 3.14 in [10]),

$$
\Lambda\left(g H g^{-1}\right) \cap \Lambda(H)=\Lambda\left(g H g^{-1} \cap H\right) .
$$

Hence, it concludes the proof by using lemma 5.4.

Now we can show proposition [5.3. Since we assume that $\psi$ is not a homeomorphism, there exists $g \in G$ such that the interior of $P$ and the interior of $g(P)$ intersect transversally. Then from lemma 5.5. $\Lambda\left(g H g^{-1}\right) \cap \Lambda(H)$ is empty. Therefore, the axis of $V$ cuts $g(P)$ transversally.

Now we assume that $c_{0}$ is smaller than the Margulis constant. Then the interior of $g(P)$ cut the Margulis tube $T$ with radius $r$ along the axis of $V$ transversally. Hence now we have a geodesic disk $\Delta=T \cap g(P)$ on $g(P)$. 
Lemma 5.6. The hyperbolic area of $\Delta$ is bigger than $4 \pi \sinh ^{2}(r / 2)$.

Proof. If $g(P)$ intersects the axis of $V$ orthogonally, then $\Delta$ is a hyperbolic disk of radius $r$; hence, the hyperbolic area of it is $4 \pi \sinh ^{2}(r / 2)$. If $g(P)$ intersects the axis of $V$ non-orthogonally, then $\Delta$ contains a hyperbolic disk of radius $r$; hence, the hyperbolic area of $\Delta$ is bigger than $4 \pi \sinh ^{2}(r / 2)$.

Now we can give a proof of theorem 5.2, By the Margulis lemma, $\Delta$ projects into the image of $g(P)$ in $\mathbf{H}^{3} / G(q)$ injectively, whereas the image of $g(P)$ in $\mathbf{H}^{3} / G$ has its hyperbolic area $2 \pi$ since it is the isometric image of a punctured cylinder. Therefore, if the hyperbolic length of $\gamma$ in $\mathbf{H}^{3} / G(q)$ is sufficiently small, we can take a radius $r$ of the Margulis tube $T$ satisfying $4 \pi \sinh ^{2}(r / 2) \geq 2 \pi$, which is a contradiction. This concludes the theorem.

5.2. A lower bound of $c_{0}$. Following [12, we have a formula of the radius of a Margulis tube.

Proposition 5.7 (cf. theorem in section 3 of [12]). For $q \in \mathcal{Q F}$, assume that $V \in G(q)$ representing a simple closed geodesic $\gamma$ in $\mathbf{H}^{3} / G(q)$, which is purely hyperbolic. If the hyperbolic length $\lambda_{\gamma}$ of $\gamma$ satisfies $\cosh \lambda_{\gamma}<\sqrt{2}$, then there is a Margulis tube with radius $r$ satisfying

$$
\sinh ^{2}(r)=\frac{1}{2}\left(\frac{\sqrt{3-2 \cosh \lambda_{\gamma}}}{\cosh \lambda_{\gamma}-1}-1\right) .
$$

The inequality $4 \pi \sinh ^{2}(r / 2) \geq 2 \pi$ and the above formula give us a lower bound of $c_{0}$.

Corollary 5.8. $\cosh ^{-1} \frac{48+5 \sqrt{2}}{49} \approx 0.493 \leq c_{0}$.

\section{EXISTENCE OF NON-STANDARD COMPONENTS}

Theorem 6.1. There is some positive constant $c_{1}$ such that for any $c$ satisfying $c>c_{1}, \mathcal{L}_{c}$ contains non-standard components.

To prove this theorem, we use the Earle slice $\mathcal{E}$ of punctured tori studied in [6, 8]. This idea is due to Raquel Diaz. We review notation of $\mathcal{E}$ (cf. [8]). The Earle slice $\mathcal{E}$ of $\mathcal{Q F}$ is the set of $G(q)=\langle A, B\rangle$ satisfying the following symmetry; there exists an elliptic element of order 2 such that $E A E=B$. Then $\mathcal{E}$ is a holomorphic slice of $\mathcal{Q F}$ and considering the conformal structure of $\Omega_{+}(G(q)) / G(q)$, it is naturally isomorphic to the Teichmüller space of punctured tori. Any element of $\mathcal{E}$ can be represented by the following matrices in $S L(2, \mathbf{C})$ of the form $A=A_{d}, B=B_{d}, d \in$ $\mathbf{C}-\{0\}$, where

$$
A_{d}=\left(\begin{array}{cc}
\frac{d^{2}+1}{d} & \frac{d^{3}}{2 d^{2}+1} \\
\frac{2 d^{2}+1}{d} & d
\end{array}\right), B_{d}=\left(\begin{array}{cc}
\frac{d^{2}+1}{d} & -\frac{d^{3}}{2 d^{2}+1} \\
-\frac{2 d^{2}+1}{d} & d
\end{array}\right) .
$$

The complex parameter $d$ gives a holomorphic embedding of $\mathcal{E}$ into the right half plane $\mathbf{C}^{+}$and we assume that $\mathcal{E}$ is embedded in $\mathbf{C}^{+}$. Then $\mathcal{E}$ contains the positive real line $\mathbf{R}^{+}$which is the Fuchsian locus $\mathcal{E} \cap \mathcal{F}$ of $\mathcal{E}$. Put $\mathbf{C}_{d^{\prime}}^{+}=\left\{d \in \mathbf{C}^{+} \mid \Re d>d^{\prime}\right\}$. To show theorem 6.1] we need lemmas. 
Lemma 6.2. There is a positive constant $d_{0}$ such that for any $d^{\prime}>d_{0}$, the hyperbolic locus $\mathcal{H}_{2 / 1}$ of $T_{2 / 1}(d)=\operatorname{Tr} W_{2 / 1}(d)$ satisfies

$$
\left(\mathcal{H}_{2 / 1}-\mathbf{R}^{+}\right) \cap \mathbf{C}_{d^{\prime}}^{+} \neq \emptyset .
$$

Proof. We remark that $W_{2 / 1}=A^{-2} B$. Then we can check our claim by direct calculation.

Lemma 6.3. There is a positive constant $d_{1}$ such that the $2 / 1$-pleating variety $\mathcal{P}_{2 / 1}$ satisfies

$$
\mathcal{P}_{2 / 1} \cap \mathcal{E} \subset \mathbf{C}^{+}-\mathbf{C}_{d_{1}}^{+} .
$$

Proof. In [8], it is shown that $\mathcal{P}_{p / q} \cap \mathcal{E}$ is equal to two components of $\mathcal{H}_{p / q}-\mathbf{R}^{+}$ terminating to the unique critical point of $\operatorname{Tr} W_{p / q}$ on $\mathbf{R}^{+}$(cf. theorem 5.1 in [8]). Then we can check our claim by direct calculation.

Lemma 6.4 (cf. [6]). There is a positive constant $d_{2}$ such that

$$
\mathbf{C}_{d_{2}}^{+} \subset \mathcal{E} .
$$

Now we can prove theorem 6.1. There is a positive constant $c_{1}$ such that for any $c>c_{1}$, there is $d \in \mathcal{E}$ such that the word $A^{-2} B$ is purely hyperbolic and $\lambda_{W_{2 / 1}}(d)=c$, but $d$ is not contained in $\mathcal{P}_{2 / 1}$. This concludes the theorem.

Remark 6.5. To estimate $c_{1}$, we need to know the size of the round disk contained in $\mathcal{E}$ tangent to the boundary $\partial \mathcal{E}$ of $\mathcal{E}$ at the origin (see [6]).

Comparing with the results in sections 5 and 6 , we have the following conjecture supported by numerical experiences by the second author.

Conjecture 6.6. There exists a unique $c_{0}$ such that $\mathcal{L}_{c}$ coincides with the standard component for any $c \leq c_{0}$, while $\mathcal{L}_{c}$ contains infinitely many non-standard components for any $c>c_{0}$.

\section{SCALING PROPERTY OF $\mathcal{L}_{c}$}

In the final section we will study the self-similar phenomena of $\mathcal{L}_{c}$ which we can observe from figures of $\mathcal{L}_{c}$ in this paper. First we remark that $\mathcal{L}_{c}$ has analytic automorphisms coming from Dehn twists.

Proposition 7.1. $(A, B) \in \mathcal{L}_{c}$ implies $\left(A, A^{n} B\right) \in \mathcal{L}_{c}$ for all $n \in \mathbf{Z}$.

Proof. The automorphism of $G$ defined by $(A, B) \mapsto\left(A, A^{n} B\right)$ is a Dehn twist along $A$ which preserves $\mathcal{Q F}$ and $\mathcal{Q}_{\gamma, c}$. $\mathcal{L}_{c}$.

The next result is easy to prove, but it induces the asymptotic self-similarity of

\section{Proposition 7.2.}

$$
\lim _{n \rightarrow \infty} \frac{\operatorname{Tr} A^{n} B}{\operatorname{Tr} A^{n-1} B}=\operatorname{Tr} A \cdot \frac{1+\sqrt{1-\left(\frac{2}{\operatorname{Tr} A}\right)^{2}}}{2},
$$

which is the attractive fixed point of the map $\operatorname{Tr} A-\frac{1}{z}$. 

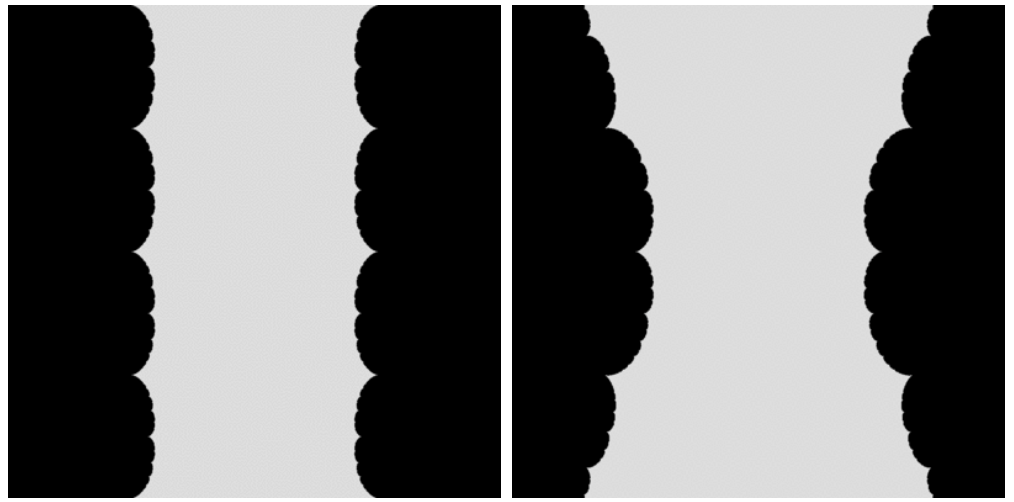

Figure 1. Maskit slice (left) and $\operatorname{Tr} A=2.5$ slice (right)
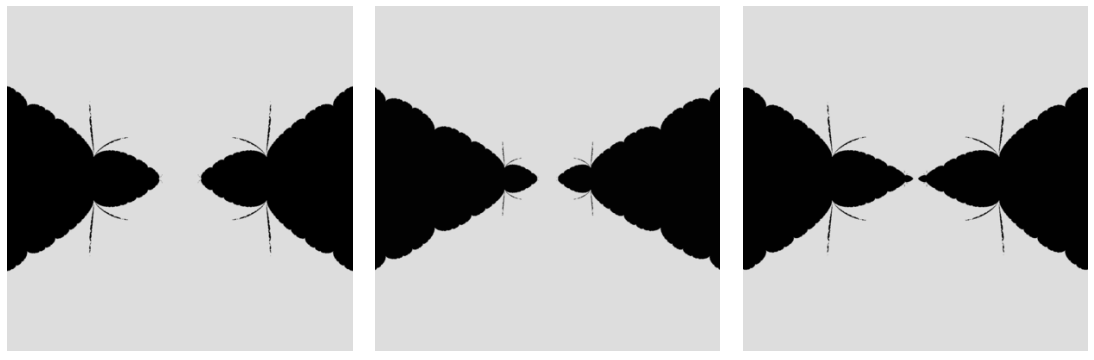

Figure 2. $\operatorname{Tr} A=8$ linear slices with ranges 16 (left), 32 (center), and 128 (right)
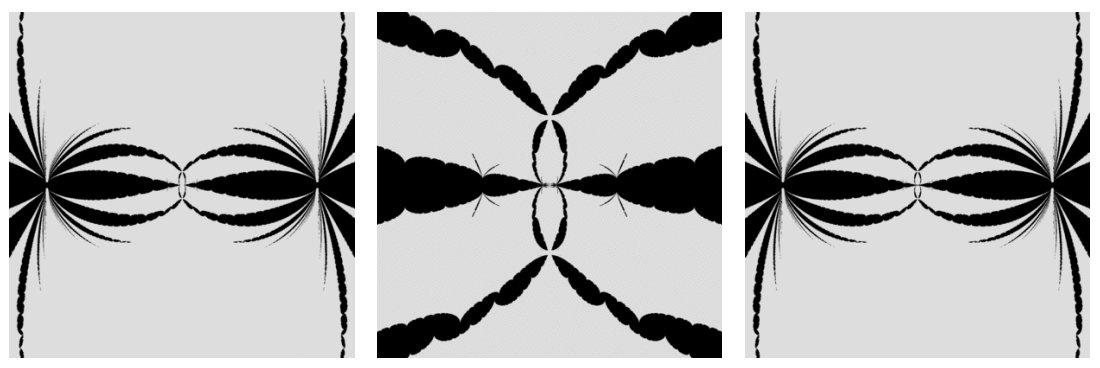

Figure 3. $\operatorname{Tr} A=100$ linear slices with ranges 128 (left), 2560 (center), and 12800 (right)

Proof. The following trace identity is well known:

$$
\operatorname{Tr} A^{n} B=\operatorname{Tr} A \cdot \operatorname{Tr} A^{n-1} B-\operatorname{Tr} A^{n-2} B .
$$

Divide both sides by $(\operatorname{Tr} A)^{n}$ and put $x_{n}:=\frac{\operatorname{Tr} A^{n} B}{(\operatorname{Tr} A)^{n}}$. Then we have

$$
x_{n}=x_{n-1}-\frac{1}{(\operatorname{Tr} A)^{2}} x_{n-2} .
$$


Moreover, put $y_{n}:=\frac{x_{n}}{x_{n-1}}$, then

$$
y_{n}=1-\frac{1}{(\operatorname{Tr} A)^{2}} \frac{1}{y_{n-1}} .
$$

Finally, put $z_{n}:=\operatorname{Tr} A \cdot y_{n}$, then

$$
z_{n}=\operatorname{Tr} A-\frac{1}{z_{n-1}} .
$$

Since $A$ is purely hyperbolic, the linear fractional transformation

$$
w=\operatorname{Tr} A-\frac{1}{z}
$$

is also purely hyperbolic; hence, all points besides the repelling fixed point of $A$ converge to the attracting fixed point of $A, \operatorname{Tr} A \cdot \frac{1+\sqrt{1-\left(\frac{2}{\operatorname{Tr} A}\right)^{2}}}{2}$. From the above arguments, $z_{n}=\frac{\operatorname{Tr} A^{n} B}{\operatorname{Tr} A^{n-1} B}$ converges to this point.

Corollary 7.3. Linear slice has an asymptotic scaling constant $\operatorname{Tr} A \cdot \frac{1+\sqrt{1-\left(\frac{2}{\operatorname{Tr} A}\right)^{2}}}{2}$.

Remark 7.4. When $A$ tends to be parabolic,

$$
\lim _{n \rightarrow \infty} \frac{\operatorname{Tr} A^{n} B}{\operatorname{Tr} A^{n-1} B}=1,
$$

which relates to the fact that the Maskit slice is invariant under translations.

Remark 7.5. Even if $A$ is loxodromic, $\mathcal{L}_{c}$ has this scaling property. Hence, we can also see that figure 10 in [11] also has such scaling property.

\section{ACKNOWLEDGMENTS}

We are grateful to Caroline Series for showing us the preprint [14 of Otal, and to Raquel Diaz for explaining to us her idea in section 6. It was fruitful for us to have discussions with them in Nara in January, 2000; in practice this work was almost done during their stay in Japan. We also wish to thank Hideki Miyachi for enjoyable conversations with him on the topic in section 7, and John Parker, Richard Canary, Kentaro Ito and Sara Maloni for telling us of their interests in our paper.

\section{REFERENCES}

1. Hirotaka Akiyoshi, Makoto Sakuma, Masaaki Wada, and Yasushi Yamashita, Punctured torus groups and 2-bridge knot groups. I, Lecture Notes in Mathematics, vol. 1909, Springer, Berlin, 2007. MR2330319(2008e:57001)

2. R. D. Canary, D. B. A. Epstein, and P. L. Green, Notes on notes of Thurston, Fundamentals of hyperbolic geometry: selected expositions, London Math. Soc. Lecture Note Ser., vol. 328, Cambridge Univ. Press, Cambridge, 2006, with a new foreword by Canary, pp. 1-115. MR2235710

3. Linda Keen and Caroline Series, Pleating coordinates for the Maskit embedding of the Teichmüller space of punctured tori, Topology 32 (1993), no. 4, 719-749. MR.1241870 (95g:32030)

4. How to bend pairs of punctured tori, Lipa's legacy (New York, 1995), Contemp. Math., vol. 211, Amer. Math. Soc., Providence, RI, 1997, pp. 359-387. MR.1476997 (98m:30063)

5. , Pleating invariants for punctured torus groups, Topology 43 (2004), no. 2, 447-491. MR2052972(2005f:30077) 
6. Yohei Komori, On the boundary of the Earle slice for punctured torus groups, Kleinian groups and hyperbolic 3-manifolds (Warwick, 2001), London Math. Soc. Lecture Note Ser., vol. 299, Cambridge Univ. Press, Cambridge, 2003, pp. 293-304. MR2044555 (2005i:57019)

7. Yohei Komori and Jouni Parkkonen, On the shape of Bers-Maskit slices, Ann. Acad. Sci. Fenn. Math. 32 (2007), no. 1, 179-198. MR2297885 (2007m:30054)

8. Yohei Komori and Caroline Series, Pleating coordinates for the Earle embedding, Ann. Fac. Sci. Toulouse Math. (6) 10 (2001), no. 1, 69-105. MR1928990(2004b:32021)

9. Christos Kourouniotis, Complex length coordinates for quasi-Fuchsian groups, Mathematika 41 (1994), no. 1, 173-188. MR1288062 (96g:30079)

10. Katsuhiko Matsuzaki and Masahiko Taniguchi, Hyperbolic manifolds and Kleinian groups, Oxford Mathematical Monographs, The Clarendon Press, Oxford University Press, New York, 1998, Oxford Science Publications. MR1638795 (99g:30055)

11. Curtis T. McMullen, Complex earthquakes and Teichmüller theory, J. Amer. Math. Soc. 11 (1998), no. 2, 283-320. MR1478844 (98i:32030)

12. Robert Meyerhoff, A lower bound for the volume of hyperbolic 3-manifolds, Canad. J. Math. 39 (1987), no. 5, 1038-1056. MR918586 (88k:57049)

13. Yair N. Minsky, The classification of punctured-torus groups, Ann. of Math. (2) 149 (1999), no. 2, 559-626. MR 1689341 (2000f:30028)

14. Jean-Pierre Otal, Sur le coeur convexe d'une variété hyperbolique de dimension 3, Preprint.

15. John R. Parker and Jouni Parkkonen, Coordinates for quasi-Fuchsian punctured torus spaces, The Epstein birthday schrift, Geom. Topol. Monogr., vol. 1, Geom. Topol. Publ., Coventry, 1998, pp. 451-478 (electronic). MR 1668328 (2000d:30065)

16. Ser Peow Tan, Complex Fenchel-Nielsen coordinates for quasi-Fuchsian structures, Internat. J. Math. 5 (1994), no. 2, 239-251. MR.1266284(94m:32030)

17. William P. Thurston, The geometry and topology of three-manifolds, Princeton lecture notes, 1980 .

Advanced Mathematical Institute and Department of Mathematics, Osaka City UniVERSITY, 558-8585, OSAKA, JAPAN

E-mail address: komori@sci.osaka-cu.ac.jp

Department of Information and Computer Sciences, Nara Women's University, 6308506 NARA, JAPAN

E-mail address: yamasita@ics.nara-wu.ac.jp 